\title{
DEVELOPMENT OF A MEASURING INSTRUMENT FOR RAPID DETECTION OF CADMIUM IONS IN WATER ENVIRONMENT
}

\author{
YANG, P. ${ }^{1,2,3}-$ SHI, J. H. ${ }^{1,2,3}-$ YU, Y. G. ${ }^{1,2,3}-$ SUN, M. ${ }^{1,2,3 *}$ \\ ${ }^{1}$ College of Information and Electrical Engineering, China Agricultural University, \\ Beijing 100083, China \\ ${ }^{2}$ Key Laboratory of Agricultural Information Acquisition Technology, Ministry of Agriculture, \\ Beijing 100083, China \\ ${ }^{3}$ Beijing Engineering and Technology Research Center for Internet of Things in Agriculture, \\ Beijing 100083, China \\ *Corresponding author \\ e-mail: sunming@cau.edu.cn; phone: +86-135-2055-7807
}

(Received $1^{\text {st }}$ Mar 2019; accepted 21 $1^{\text {st }}$ May 2019)

\begin{abstract}
In this study, heavy metal cadmium ions $\left(\mathrm{Cd}^{2+}\right)$ in water environment were studied, and the existing $\mathrm{Cd}^{2+}$ colorimetric system was simplified. Based on colorimetry and spectroscopy, a portable analyzer for rapid detection of $\mathrm{Cd}^{2+}$ was developed. This study simplifies the tedious and complex preprocessing and completes the colorimetric experiment. The measuring instrument is mainly divided into an optical path module and a circuit module. The optical path module converts the concentration of cadmium ions into an optical signal, and the circuit module realizes functions of converting, amplifying, data processing, viewing management data, and uploading data of the optical signal. After the detector was completed, several system performance analyses were performed, and low power consumption, antiinterference, and repeatability tests were taken to verify the accuracy of the measurement. The experimental results show that the determination coefficient of the model and the chemical value of the colorimetric reaction is $\mathrm{R}^{2}=0.934$, the detection limit is $0.1047 \mathrm{mg} / \mathrm{L}$, the detection range is $0.1 \sim 1 \mathrm{mg} / \mathrm{L}$, and the variation coefficient of cadmium content index is less than $1.0 \%$. The test results show that the tester has good accuracy and repeatability. At the same time, compared with other related instruments, the meter also has the advantages of multiple functions, small size, portability, convenient use, and low power consumption.
\end{abstract}

Keywords: cadmium ion, colorimetry, spectroscopy, analyzer, rapid detection

\section{Introduction}

With the rapid development of modern industry, the danger of heavy metal pollution has become increasingly prominent. Cadmium is a highly toxic heavy metal and is a non-essential toxic element of the human body. It is called the "first of the five poisons" (Bairi et al., 2014; Chansuvarn, 2012). As early as 1974, the United Nations Environment Programme (UNEP) and the International Committee for Work Health and Heavy Metals identified it as a key pollutant . Numerous studies have shown that cadmium is carcinogenic, and the International Agency for Research on Cancer (IARC) classifies cadmium as the first class of human carcinogens (Chen et al., 2014; Chen et al., 2015). Wastewater containing cadmium is discharged into the environment. Cadmium has a strong enrichment effect in aquatic animals. Fish can be enriched 100,000 times. Once absorbed by the human body through the food chain, cadmium will selectively accumulate liver and kidney (Kim et al., 2012). Cadmium can also hinder the metabolism of bones, which is the main culprit for "pain sickness" (Fang, 
2016). Therefore, it is especially important to study the efficient detection of cadmium ion concentration.

The current detection methods are: atomic emission spectroscopy, mass spectrometry, fluorescence analysis (Georgiev et al., 2014; Ghasemi, 2009), electrochemical analysis method (Han, 2013), spectrophotometry (Mohanty et al., 2018), atomic absorption spectrometry ( Han et al., 2014; HJ-763, 2015). Atomic absorption spectroscopy (Huang et al., 2013; Huang et al., 2012) overcomes the shortcomings of the traditional method, which has the advantages of low detection line, easy operation, and fast analysis speed (Kai, 2015) However, the range of atomic absorption bands is narrow, and it is difficult to analyze complex samples. Spectrophotometry is simpler to choose from, simple and fast to operate, and has a wider range of applications, but it is relatively low in accuracy. Electrochemical analysis methods ( $\mathrm{Li}, 2006$ ), although the detection efficiency is high, the accuracy accuracy is limited. Mass spectrometry (Li, 2008;Mohan et al. ,2018) can quickly detect metal concentration on the basis of a small amount of reagents (Rajalakshmi, 2011;Banerjee et al. ,2008), but it has limitations in the field of organic mass spectrometry. The application of various methods provides different ideas for cadmium ion detection. (Wanget al. , 2003; Ying ,2013)

In recent years, there have been many research results for the measurement of cadmium ion colorimetry. Yuan Aiping used dry ashing to dissolve ash with warm 0.2 $\mathrm{mol} / \mathrm{L}$ sulfuric acid, and added dithizone-carbon tetrachloride as a masking agent to eliminate copper interference in the matrix and used for cadmium in fish and meat foods. The detection limit of the method was $0.12 \mu \mathrm{g} / \mathrm{L}$, and the recovery was $86 \%$ $112 \%$. Fan Yueqin et al. studied the color reaction of the new reagent 4-methyl-2,5disulfonylphenyldiazoaminoazobenzene with cadmium . In the presence of TritonX100 , the reagent forms a red complex with cadmium in a buffer solution of $\mathrm{Na}_{2} \mathrm{~B}_{4} \mathrm{O}_{7}-$ $\mathrm{NaOH}$ at $\mathrm{pH}$ 10.5 11.5. The maximum absorption wavelength is $526 \mathrm{~nm}$ and the apparent molar absorptivity is $2.5 \times 105 \mathrm{~L} \cdot \mathrm{mol}^{-1} \cdot \mathrm{cm}^{-1}$. This study combines colorimetry and spectroscopy to simplify the complex and cumbersome pre-processing of colorimetric methods, transform $\mathrm{Cd}^{2+}$ into measurable compounds, construct detection models, and apply the model to portable spectrometers to develop low cost. Low detection limit, simple pre-treatment, suitable for a variety of heavy metal rapid detection instrument.

\section{Materials and methods}

\section{Materials}

The heavy metal cadmium ion $\left(\mathrm{Cd}^{2+}\right)$ was used as the research object. The test materials included dithizone, carbon tetrachloride, sodium tartrate, sodium hydroxide, hydroxylamine hydrochloride and distilled water. The instruments used are $\mathrm{pH}$ meters, beakers, electronic balances, centrifuge tubes, and the like. The test principle is that in a strong alkali sodium hydroxide solution, $\mathrm{Cd}^{2+}$ in the water sample can react with dithizone to form a red complex.

In the colorimetric experiment, dithizone was used as a color developer, and the solvent was carbon tetrachloride $\left(\mathrm{CCl}_{4}\right)$. In the experiment, sodium potassium tartrate solution was used as a masking agent to exclude magnesium, iron, calcium and aluminum ions to prevent these ions from forming hydroxide precipitates in an alkaline environment (Alfonso,2010; Zhang et al,2010). The colorimetric reaction can be carried 
out under a strong alkaline environment and adjusted with a sodium hydroxide solution. As a color developing agent, dithizone has a molecular formula of $\mathrm{C}_{13} \mathrm{H}_{12} \mathrm{~N}_{4} \mathrm{~S}$, a blueblack crystalline powder, which is easily oxidized by air and can be protected by a hydroxylamine hydrochloride solution. (Zhang et al, 2015;Zhao et al,2011) Carbon tetrachloride is a colorless solution with large specific gravity, low viscosity, nontoxicity and good chemical stability. It is selected as a solvent for dithizone solution. After the dithizone is dissolved in carbon tetrachloride, the solution is blue-green, and the red complex formed by the colorimetric reaction with cadmium is obviously different, which meets the requirements of the colorimetric reaction.(Wu et al, 2015; Sponza et al,2011)

\section{Methods}

The specific test steps are as follows:

Solution preparation: test to simulate cadmium-containing water, prepare 66 gradients of cadmium solution from 0.001 to $1 \mathrm{mg} / \mathrm{L}$ with standard solution; configure $1 \mathrm{mg} / \mathrm{L}$ dithizone use solution, use carbon tetrachloride as solvent; configure $20 \%$ The hydroxylamine hydrochloride solution is used as a reducing medium; $10 \%$ sodium potassium tartrate solution is used as a masking agent; the colorimetric reaction can be carried out under a strong alkaline environment, and the sodium hydroxide solution is used for adjustment.

The maximum wavelength of the absorption of the magenta liquid after the reaction was measured by a visible spectrophotometer V-1300 was $530 \mathrm{~nm}$, and did not change with the change of the concentration. Therefore, this study chose $530 \mathrm{~nm}$ as the incident light center wavelength.

The optical path portion is composed of an LED light source, a fiber optical path, and a silicon detector. The required light source band of the circuit is $530 \mathrm{~nm}$. Select the LED plus filter to achieve the required narrow-band light source to ensure the monochromaticity of the light source. The filter has relatively stable performance, high transmittance, and can be well matched to the established model. After comparison, this study selected the LED light source of model E505-06 from Japan EPETEX and the filter of $530 \mathrm{~nm}$ center wavelength of INTOR Company of USA.

In order to enable the monochromatic light source to emit parallel light and vertically illuminate the detector, and simplify the structure of the instrument, this study uses a fixed fiber collimator as the optical path, and realizes the connection between the reaction black box and the circuit, as shown in Figure 1. One end of the fiber is the incident end, and the outside is a metal sleeve, which is used to fix the filter and the LED light source, to ensure the stability and reliability of the light source, and to reduce the experimental error. The structure is shown in Figure 2. In this study, a cuvette with a diameter of $1 \mathrm{~cm}$ was used as the absorption cell to carry the sample.

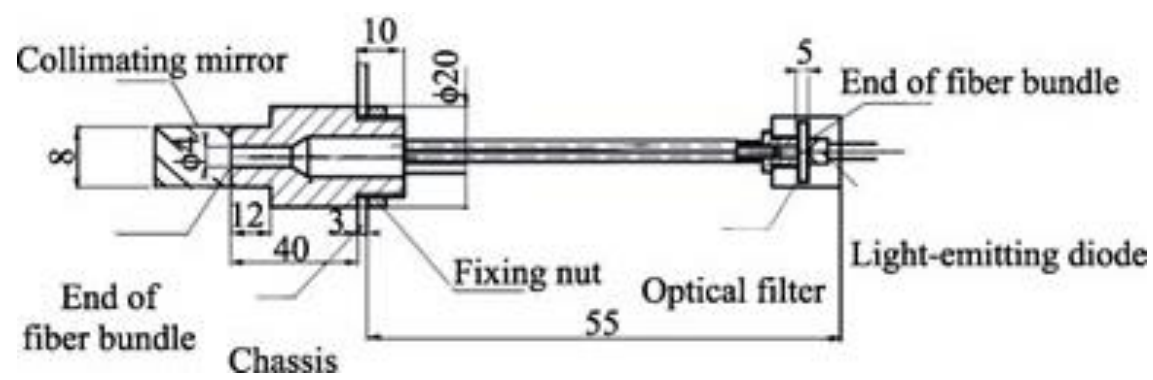




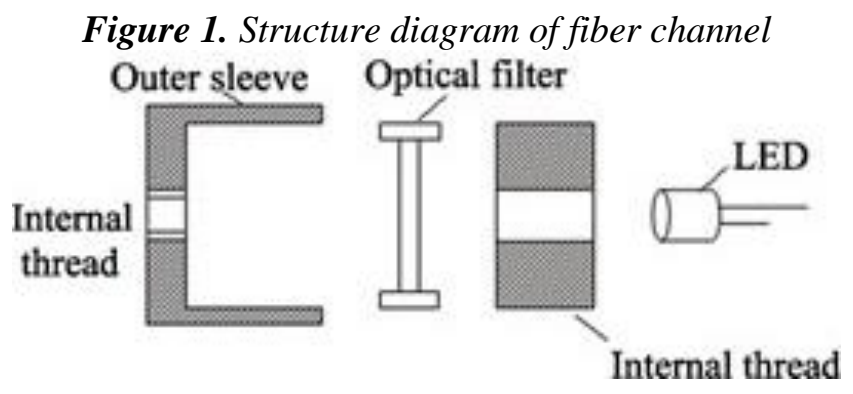

Figure 2. Schematic diagram of metal sleeve

The circuit module is mainly composed of a power supply circuit, a detection and amplification circuit, a keyboard circuit, a liquid crystal display circuit, etc., to realize conversion of an optical signal, amplification of an electric signal, attenuation of noise, and data processing and display functions. The instrument uses TI's low-power 16-bit microcontroller MSP430F149 as the controller of the system, with a 16-bit reduced instruction set to meet the rapid detection design of the analyzer.

The measuring instrument adopts a silicon material photodiode detector with a spectral response range of 300 to $1100 \mathrm{~nm}$, and the model number is PS0308 (product of FIRST SENSOR, Germany), which effectively ensures the linearity of the instrument. Since the design circuit of this study works in a low frequency environment and requires good linearity, the photodiode is selected to work in zero bias mode without dark current error, and there is no need to design a reverse voltage bias circuit.

This design adopts full dot matrix graphic liquid crystal display CSME-12864, which has the advantages of low voltage, low consumption and good backlight mode. At the same time, this design uses membrane keyboard to realize functions of viewing, measuring, flipping, deleting, storing data, etc. powered by.

\section{Results}

The partial least squares method is combined with the above four different partitioning methods, combined with the MSC pretreatment method to establish a mathematical model of cadmium ion concentration and absorbance. The model has several evaluation indicators, and the model results are intuitive and specific through specific index values. Analysis, the results are shown in Table 1.

Table 1. Comparison of different modeling set partitioning methods combined with PLS cadmium ion concentration prediction model

\begin{tabular}{c|c|c|c|c|c|c}
\hline \multirow{2}{*}{$\begin{array}{c}\text { Modeling set } \\
\text { partitioning } \\
\text { method }\end{array}$} & \multirow{2}{*}{ Main factor } & \multicolumn{2}{|c|}{ Modeling set } & \multicolumn{2}{|c|}{ Forecast set } & \multirow{2}{*}{ RPD } \\
\cline { 3 - 6 } & & Rc & RMSECV & Rp & RMSEP & \\
\hline RS & 3 & 0.9427 & 0.1094 & 0.9131 & 0.1317 & 2.5074 \\
KS & 2 & 0.9619 & 0.0792 & 0.9201 & 0.1246 & 2.6101 \\
SPXY & 4 & 0.9662 & 0.0768 & 0.7068 & 0.1850 & 1.4455 \\
Duplex & 5 & 0.9550 & 0.0970 & 0.9549 & 0.0863 & 3.6142 \\
\hline
\end{tabular}


From the analysis of Table 1, it can be known that the RS method divides the modeling set, the range does not cover all the samples, is not representative, and the established model is not stable enough. The model established by the Duplex method has the best detection and detection results. The RMSECV and RMSEP are $0.0970 \mathrm{mg} / \mathrm{L}$ and $0.0863 \mathrm{mg} / \mathrm{L}$, respectively, which are small, and are smaller than other models. The RPD value is 3.3142 over 3, indicating that the model predicts better. Therefore, Duplex is selected as the model selection method of modeling set, and the PLS model of cadmium ion concentration is established by MSC pretreatment method. The model and prediction map of the obtained model are shown in Figures 3 and 4, and the sample is evenly distributed in the curve. Left and right, achieved a good prediction effect.

The test method is to prepare a set of lead standard solution $0.001 \sim 1 \mathrm{mg} / \mathrm{L}$ a total of 66 sets of gradient solution. The measurement was carried out by using a $530 \mathrm{~nm}$ light source with a bandwidth of $10 \mathrm{~nm}$, and the linear regression analysis was obtained. The modeling results and test results are shown in Figure 3.

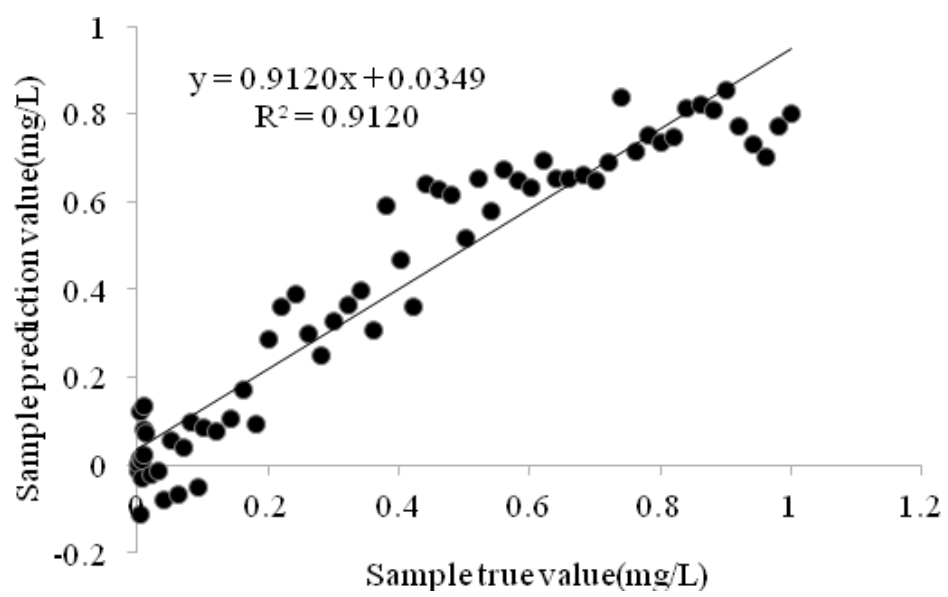

Figure 3. Correlation between the actual value of $\mathrm{Cd}^{2+}$ concentration and the modeling set of predicted values

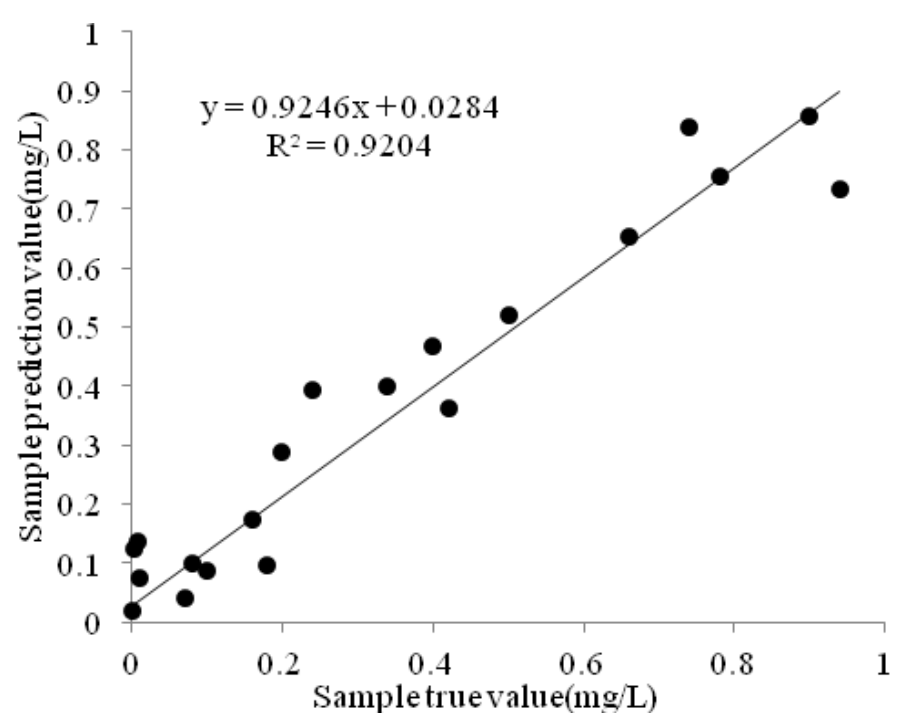

Figure 4. Correlation between the true value of $\mathrm{Cd}^{2+}$ concentration and the validation set of predicted values 
The design is based on the colorimetric reaction of dithizone with cadmium ions, masking the interference of other heavy metal ions with masking agent, and optimizing the reaction environment, including $\mathrm{pH}$, temperature and other influencing factors. The whole instrument is composed of optical path module and circuit module. The linear measurement range of the instrument is $0.1 \sim 1 \mathrm{mg} / \mathrm{L}$, which can measure the low concentration of cadmium ions and achieve a certain precision. The national surface water environmental quality standard for cadmium ions is $0.01 \mathrm{mg} / \mathrm{L}$, and the instrument meets the testing standards. The detection range of dithizone spectrophotometry in national standard is $0.01 \sim 0.3 \mathrm{mg} / \mathrm{L}$. The detection range of this method meets the standard requirements, and the experimental operation is simple. Compared with the spectrophotometer, the optical path system of the instrument is simple in design, and the main structure is composed of LED, filter and optical fiber. The measurement room has good shading performance, can shield the influence of stray light, and has simple and reliable design. The instrument passed the repeatability test with a coefficient of variation of less than $1 \%$ and hardware and software antiinterference measures to make it have certain stability. The tester has certain innovations. The actual experimental verification results show that the prototype of this design has good repeatability and stability, and can be used for the measurement of cadmium ions in water.

\section{Discussion}

By comparing the detection range of the method to meet the standard requirements, the experimental operation is simple. Compared with the spectrophotometer, the optical path system of the instrument is simple in design, and the main structure is composed of LED, filter and optical fiber. The measurement room has good shading performance, can shield the influence of stray light, and the design is simple and reliable. The instrument passed the repeatability test with a coefficient of variation of less than $1 \%$ and hardware and software anti-interference measures to make it have certain stability. The tester has certain innovations. The actual experimental verification results show that the prototype of this design has good repeatability and stability, and can be used for the measurement of cadmium ions in water.

\section{Conclusion and recommendation}

In this study, heavy metal $\mathrm{Cd}^{2+}$ was used as the research object to simplify the existing $\mathrm{Cd}^{2+}$ colorimetric system, and a portable analyzer for $\mathrm{Cd}^{2+}$ rapid detection was developed.

(1) By analyzing the physicochemical properties of $\mathrm{Cd}^{2+}$ and the colorimetric reaction of cadmium with dithizone, the experimental scheme for the color reaction of dithizone with water-weight Cadmium metal in alkaline environment at normal temperature was determined, and a masking agent was used. Mask other ion interference.

(2) The detection range of the analyzer in this study is $0.1 \sim 1 \mathrm{mg} / \mathrm{L}$, the coefficient of variation is less than $1 \%$, and the repeatability test results are good. At the same time, the analyzer has simple and stable structure, low power consumption and portability. Further research on this analyzer can be carried out in future work to achieve rapid detection of various heavy metals in the actual water environment. 
Acknowledgements. Fund for this research is provided by Development of the decision-making system in agricultural non-point source and heavy metal pollution management (No.2016YFD0800907).

\section{REFERENCES}

[1] Alfonso, M., Tarraga, A., Molina, P. (2010): Ferrocene-based multichannel molecular chemosensors with high selectivity and sensitivity for $\mathrm{Pb}$ (II) and $\mathrm{Hg}(\mathrm{II})$ metal cations. Dalton Transactions 39(37): 8637-8645.

[2] Bairi, P., Chakraborty, P., Roy, B. (2014): Sensing of $\mathrm{Hg}^{2+}$, and $\mathrm{Ag}^{+}$, through a $\mathrm{pH}$ dependent FRET system: fabrication of molecular logic gates. - Sensors \& Actuators B Chemical 193(3): 349-355 (in Chinese with English abstract).

[3] Banerjee, S., Kara, S., Santra, S. (2008): A simple strategy for quantum dot assisted selective detection of cadmium ions. - Chemical Communications 26: 3037-3039.

[4] Chansuvarn, W., Imyim, A. (2012): Visual and colorimetric detection of mercury(II) ion using gold nanoparticles stabilized with a dithia-diaza ligand. - Microchimica Acta 176(1): 57-64.

[5] Chen, G. H., Chen, W. Y., Yen, Y. C. (2014): Detection of mercury (II) ions using colorimetric gold nanoparticles on paper-based analytical devices. - Analytical Chemistry 86(14): 6843-6849.

[6] Chen, Z., Zhang, C., Tan, Y. (2015): Chitosan-functionalized gold nanoparticles for colorimetric detection of mercury ions based on chelation-induced aggregation. Microchimica Acta 182(3): 611-616.

[7] Fang, Y. J., Xia, D. Z. (2016): Determination of cadmium ion binding ability of natural product extracts by spectrophotometry. - Chinese Journal of Traditional Chinese Medicine and Pharmacy 2: 295-298.

[8] Georgiev, N. I., Asiri, A. M., Qusti, A. H. (2014): Design and synthesis of pH-selective fluorescence sensing PAMAM light-harvesting dendrons based on 1,8-naphthalimides. Sensors \& Actuators B Chemical 190(1): 185-198.

[9] Ghasemi, J. B., Zohrabi, P. (2009): Spectrophotometric complexation study of 4-(2Thiazolylazo) Resorcinol and $\mathrm{Zn}^{2+}, \mathrm{Cd}^{2+}, \mathrm{Pb}^{2+}$ and $\mathrm{Cu}^{2+}$ in $50 \%$ DMSO/water solution. Chemia Analityczna 54(5): 1035-1049.

[10] Han, X. (2013): Detection of Heavy Metal Cadmium in Soil Based on Reagent Colorimetric and Spectral Analysis. - China Agricultural University, Beijing.

[11] Han, Z. X., Zhu, B. S., Wu, T. L., Yang, Q. Q., Xue, Y. L., Zhang, Z.,Wu, X. Y.(2014): A fluorescent probe for $\mathrm{Hg}^{2+}$, sensing in solutions and living cells with a wide working pH range. - Chinese Chemical Letters 25(1): 73-76 (in Chinese with English abstract).

[12] HJ-763 (2015): Specifications and test procedures for automatic monitoring of cadmium in water.

[13] Huang, J., Li, M. X., Liang, X. F. (2013): Rapid determination of lead in tin alloy jewelry by dithizone colorimetric method. - Journal of Inspection and Quarantine 2: 5-8.

[14] Huang, X. W., Zhao, Z. X., Li, H. S., Wang, F. S. (2012): Determination of trace mercury by ultrasonic anodic stripping voltammetry. - Chemical Reagents 34(6): 532-534.

[15] Kai, Y. (2015): Determination of trace cadmium in soil by complex spectrophotometry. Trace Elements and Health Research 32(1): 66-67.

[16] Kang, R. Q., Yang, S., Wang, Y. L. (2014): Determination of cadmium in cosmetics in a city by flame atomic absorption spectrophotometry. - Chemical Engineer 28(12): 21-23.

[17] Kim, H. N., Ren, W. X., Kim, J. S. (2012): Fluorescent and colorimetric sensors for detection of lead, cadmium, and mercury ions - Chemical society reviews 41(8):32103244.

[18] Kim, I. B., Bunz, U. H. (2011): Modulating the sensory response of a conjugated polymer by proteins: an agglutination assay for mercury ions in water. - Journal of the American Chemical Society 128(9): 2818-2819 
[19] Li, M. Z. (2006): Spectral Analysis Technology and Its Application. - Science Press, Beijing.

[20] Li, Y. Q. (2008): Progress in the application of atomic absorption spectrometry in the analysis of heavy metal cadmium and cadmium. - Metallurgical Analysis 28(6): 33-41.

[21] Mohan, B. K., Padhy, R., Mohapatra, M., Sabat, G., Behera, S. K., Jena, S., Behera, J. K. (2018): Physio-Chemical analysisi of wastewater samples from drainage systems of Berhampur muncipality area of Odisha. - International Journal of Plant, Animal and Environmental Sciences 8(4): 1-5.

[22] Rajalakshmi, K., Haribabu, T. E., Sudha, P. N. (2011): Toxic kinetics of antioxidants from P. tricornutum and calendula exposed to heavy metal lead. - International Journal of Plant, Animal and Environmental Sciences 1(2): 105-109.

[23] Sponza, D., Karaogiu, N. (2002): Environmental geo chemistry and pollution studies of Aliage metal industry district.- Environment International 27:541-553.

[24] Wang, Z. K., Xu, J. W., Guo, M. (2003): Research progress in detection technology of heavy metal cadmium ions. - 68(486): 167-82.Xu, S. X., Wang, B. (2016): Determination of cadmium residues in vegetables by dithizone method. - Anhui Agricultural Sciences 44(14): 109-110.

[25] Wu, F., Mao, M., Cen,Y. (2017): Copolymerization of Eu(TTA) 3Phen doped styrene and methyl methacrylate nanoparticles and use in quantitative detection of pepsinogen - Rsc Advances 702(1): 102-108.

[26] Ying, H. J. (2013): Research and Development of Heavy Metal Ion Sensors. - Jiangnan University, Wuxi.

[27] Zhang, G., Zhao, G. Q., Jiang, J. J. (2010): Study on enzymatic detection of cadmium ions in foods. - Food Engineering: 51-53.

[28] Zhang, Z. H., Wang, Z. Q., Wang, H., Duan, Y. Y., Liu, G. (2015): Design and experiment of rapid detection system for soil cadmium element. - Journal of China Agricultural University 20(1): 157-163.

[29] Zhao, H., Nan. T., Tan, G. (2011): Development of two highly sensitive immunoassays for detection of copper ions and a suite of relevant immunochemicals - Analytica Chimica acta 702(1):102-108. 